\title{
A High Performance 6-DOF Haptic Cobot
}

\author{
Eric L. Faulring, J. Edward Colgate and Michael A. Peshkin \\ Department of Mechanical Engineering \\ Northwestern University \\ Evanston, IL 60208 \\ e-faulring@northwestern.edu
}

\begin{abstract}
A novel, six-degree-of-freedom active haptic device is introduced. A parallel kinematic design and the use of nonholonomic continuously variable transmissions provide extremely high stiffness in directions that would violate a virtual constraint. At the same time, smooth motion is permitted tangential to virtual constraints and in open space. High quality constraint surfaces having one to five dimensions can be displayed. A notable feature of this device is the mutual coupling of all six linear actuators to a common rotating cylinder, which can, optionally, be powered. The resulting mechanism is simple to control, and allows several new control strategies in Cobotic haptics.
\end{abstract}

Keywords - cobot; haptic; teleoperation; parallel; nonholonomic; admittance; impedance;

\section{INTRODUCTION}

\section{A. Motivation}

There are an increasing number of tele-operation based tasks in which a high fidelity haptic master controller is desired. These include the control of a slave robot in medical surgeries, micro/nano-manipulation, undersea salvage, regular maintenance of nuclear plants and other hazardous environments, as well as decontamination and decommissioning of many chemical and nuclear facilities. The execution of these tasks by a remote operator is affected by his/her sense of tele-presence and the transparency of the master-slave relationship[1]. This illusion of presence is enhanced by audio, visual and haptic cues. While visual cues are certainly mandatory, and audio a benefit at times, haptic cues can significantly improve the flow of information from the slave site to the remote operator for many dexterous tasks.

The specific application for development of the master hand controller introduced in this paper is the tele-operation of the Dual Arm Work Platform (DAWP) at Argonne National Laboratory[2, 3]. The DAWP is a platform containing two sixdegree-of-freedom Schilling Manipulators, several tools and seven cameras. The DAWP can be lowered into a nuclear reactor environment to perform disassembly. The tool inventory consists of a portable band saw, circular saw, pry bar, and wrench. The manipulators are hydraulically actuated serial link robots and contain a gripper as a seventh degree of freedom that can attach rigidly to the specially outfitted tools.

The existing master controllers for the DAWP consist of two "mini-master" manipulators, which share the same kinematics as the slave manipulators, but are completely

The authors would like to acknowledge the support of the Department of Energy, grant number DE-FG07-01ER63288. passive devices, equipped only with encoders for use as input devices. For the DAWP operator, 90 percent of his/her time is spent in alignment procedures, often after slipping off the head of a bolt or out of a cut in progress[4]. A collaborative sister project at Argonne National Laboratory seeks to automate certain tasks while the development of the Cobotic Hand Controller at Northwestern seeks to improve an operator's execution of manual tasks[5].

One of the key improvements the Cobotic Hand Controller (See Figure 1) can provide to the DAWP is the implementation of virtual surfaces, or virtual constraints on motion, as suggested by Rosenberg[6]. While this could be done at the slave side in the existing system, an active master will allow the reproduction of these constraints at the master, and greatly reduce operator fatigue and increase efficiency by eliminating unneeded motions in six-space. Thus if the operator is using a saw, and constrains the motion of the saw to the plane of the blade at the slave, he/she will feel these same constraints at the master.

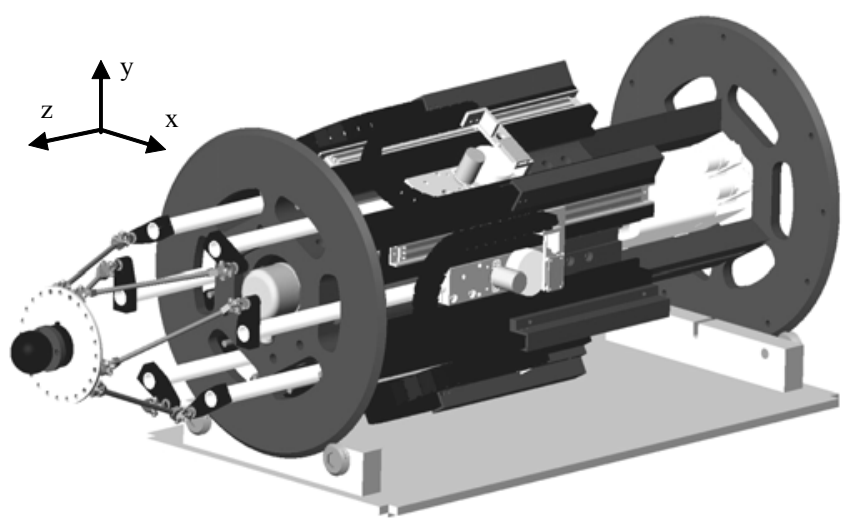

Figure 1. A CAD model of the 6-DOF Cobotic Hand Controller.

Design objectives for the Cobotic Hand Controller included recommended characteristics of a high bandwidth force reflecting manipulandum: low inertia, low friction, high stiffness, back-drivability, zero backlash, gravitational counterbalancing, and output force capabilities matched to the human operator[7].

The chief specification needed in order to represent hard realistic virtual constraints is high realizable stiffness. A search of the literature and commercial websites resulted in the following table of notable 6 or 7 degree of freedom haptic 
devices (Table I). The peak and continuous forces and torques, approximate translational and rotational workspaces, and the maximum values for structural or displayable virtual stiffness are reported where available[8-14]. The Cobotic Hand Controller's significant structural stiffness is not due to powerful actuators and will be discussed in detail later.

TABLE I. PROPERTIES OF 6 AND 7 DOF HAPTIC Displays

\begin{tabular}{|c|c|c|c|c|c|}
\hline Product & Force(N) & Torque(Nm) & Translation(cm) & Rotation(deg) & Stiffness(kN/m) \\
\hline Delta (Force Dimension) & NA,25 & NA,0.2 & $30,36,36$ & $40,40,40$ & NA \\
\hline Phantom 1.5/6DOF (Sensable) & $8.5,1.4$ & $0.5,0.2$ & $20,27,38$ & $260,335,335$ & 3.5 \\
\hline Virtuose 6D35-45 (Haption) & 35,10 & 3.1, NA & $45,45,45$ & $210,120,160$ & 2 \\
\hline Pathos II (Pohang) & 60, NA & NA,NA & $14,14,14$ & $90,95,180$ & $17-72$ \\
\hline Freedom 7 (McGill) & 5, NA & NA,NA & $13,16,18$ & $90,100,120$ & NA \\
\hline Haptic Device (Hanyang) & 20, NA & 1.0, NA & $10,10,10$ & $45,45,90$ & NA \\
\hline Modified Delta(Tohoku) & 10, NA & NA,NA & $15,15,15$ & $140,140,140$ & NA \\
\hline Cobotic Hand Controller & 50,50 & 2,2 & $17,17,17$ & $80,90,170$ & $20-400$ \\
\hline
\end{tabular}

\section{B. Cobots}

The word "cobot" is a concatenation of collaborative and robot, meaning shared control between human operator and computer. The use of non-holonomic constraints as the basis for cobot technology is best explained through previous cobots $[15,16]$. The first and simplest is the Unicycle Cobot. A steered wheel, un-powered about its rolling axis, creates a relationship between the two components of its linear velocity. More complex, is the Scooter Cobot, a platform with three steered wheels, which lives in a three-dimensional planar taskspace. A force sensor atop Scooter interprets an operator's intent, and the control algorithm steers the wheels accordingly. Also in existence is the Arm Cobot which provides a threetranslational workspace[17].

Cobots can either be operated in "free-mode," where the intent of the operator in the full dimension of the task space is followed completely, or in "virtual-surface" mode, where a lower dimensional surface than the task space guides the operator's intent tangent to that surface, and the non-holonomic constraints of the rolling wheels, not the torque of any actuators, prevent motion normal to the virtual surface. The steering motors cannot cause a passive cobot to move, and therefore the device is inherently safe. The device can defend extremely strong and stable virtual paths and constraints.

\section{Design of The Cobotic Hand CONTROLleR}

\section{A. Geometry}

The design of this 6-DOF Cobotic Hand Controller utilizes the kinematics of a parallel platform introduced by Merlet[18]. The proximal links are coupled by three degree-of-freedom universal joints to the distal links, and these in turn are coupled via two degree-of-freedom universal joints to an end-effector platform. Here a force sensor is placed to determine the user's intent. Our addition to Merlet's kinematics has been to couple the six linear actuators to a central "power cylinder" through non-holonomic constraints.

Figure 2 details the assembly of the joints between proximal and distal links, and between distal links and the endeffector. Linear actuation of the proximal links is achieved via a rotational to linear continuously variable transmission (CVT), namely a steered wheel. The angle of each wheel relates the linear velocity $v_{\mathrm{i}}$ of each proximal link to the rotational velocity of the power cylinder $\omega$. When the wheels are steered such that their rolling axis is parallel to the power cylinder $\left(\phi_{1}=0\right)$, a ratio $v_{\mathrm{i}} / \omega=-r \tan \left(\phi_{1}\right)=0$ is set. If the wheels are steered either direction from $\phi_{1}=0$, ratios between \pm infinity can be achieved. In practice, wheel slip limits this range. It is also evident, that turning all six wheels to $\phi_{1}=0$ locks the six actuators, and turning them to $\phi_{1}=\pi / 2$ completely decouples the actuators from the cylinder's velocity, although the cylinder would then be unable to turn.

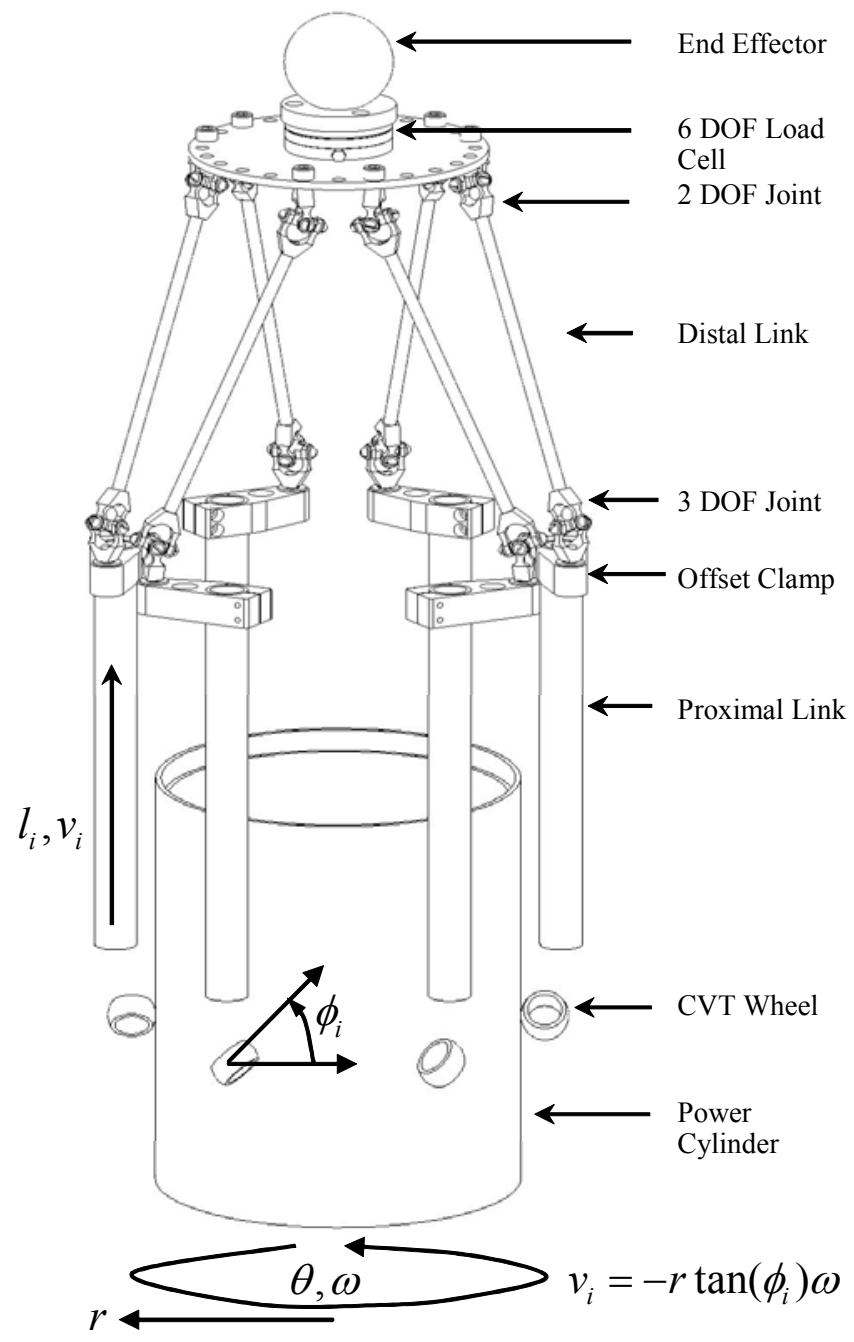

Figure 2. The kinematics of a Merlet-Cobotic parallel platform (not to scale). This design consists of six linear actuators arrayed around a central power cylinder. Figure 6 details the structure connecting the CVT wheels to the proximal links.

As it was not a design goal to optimize the kinematic geometry for near-isotropic stiffness or certain workspace requirements, the robot was designed for some degree of kinematic flexibility. Thus the offset clamps (Figure 2) adjoining proximal and distal links have two attachment points for the distal links and can be rotated about the proximal links. Rotating them inward yields a larger rotational workspace but reduced stiffness. The mounting positions of the distal universal joints to the end-effector are adjustable as well. In 
addition the length of the distal links is easily changed as they are made of threaded rod that inserts directly into the universal joints.

The universal joints themselves are remarkable in that they exhibit continuous rotation even when coupling shafts at an angle of 87 degrees, close to perpendicular. This severe operation angle would be detrimental if they needed to transmit torque, but here they need only maintain a kinematic constraint. They were designed and built specifically for the Cobotic Hand Controller and each contain four preloaded radial bearings.

\section{B. Proximal Links and Superstructure}

As shown in Figure 3, there are six equally spaced proximal links and their actuator assemblies. These assemblies, detailed in Figures 4-6, are bolted to a central core detailed in Figure 7. Actuators 1 and 2 in Figure 3 have various components removed for visibility. For actuator 1 , the linear guide way and wire management has been removed, allowing a view of the moving components. A carriage containing the CVT wheel, steering motor, rotational encoder and linear potentiometer wiper is the attachment point for the base of each proximal link. Each of these carriages can be removed independently if the proximal - distal offset clamp is detached. For actuator 2, all but the proximal link and CVT wheel have been removed, exposing the power cylinder beneath.

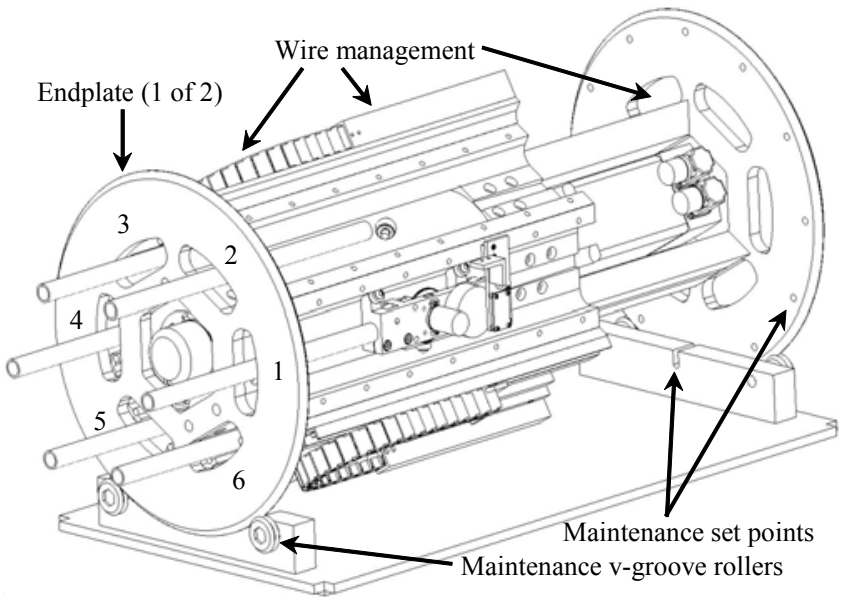

Figure 3. Six actuator assemblies for proximal links 1-6 are arrayed about a central power cylinder. Actuators 1 and 2 have selected components removed for visibility. The two ends of the device are capped by endplates which sit in v-groove rollers. Thus the whole device can be rotated and fixed at increments of 30 degrees for maintainence or kinematic purposes. Wire management guides all wiring(not shown) through the rear endplate.

\section{Six ActuatorAssemblies}

The parallel nature of the mechanism allows for six identical actuator assemblies. One of these is shown in Figure 4. All structural components are machined from aluminum with the exception of the proximal links. These are $15.875 \mathrm{~mm}$ diameter ceramic tubes due to their high strength to weight ratio and the confines of space. The current length ceramic tubes provide $24 \mathrm{~cm}$ of workspace in $\mathrm{z}$ translation. The upper limit of the workspace is limited by the cylinder's dimensions.

A conductive plastic linear potentiometer is chosen for a continuous linear sensor over numerous digital incremental options due to the lightweight and wireless wiper, as well as the ability to perform analog differentiation in order to obtain a high resolution velocity signal.

The CVT steering motors couple to their respective wheel assemblies via single stage transmissions, thus the CVT motor and CVT encoder are not co-axial (Figure 4). The transmissions employ fine-pitched gears that result in minimal backlash (Figure 6).

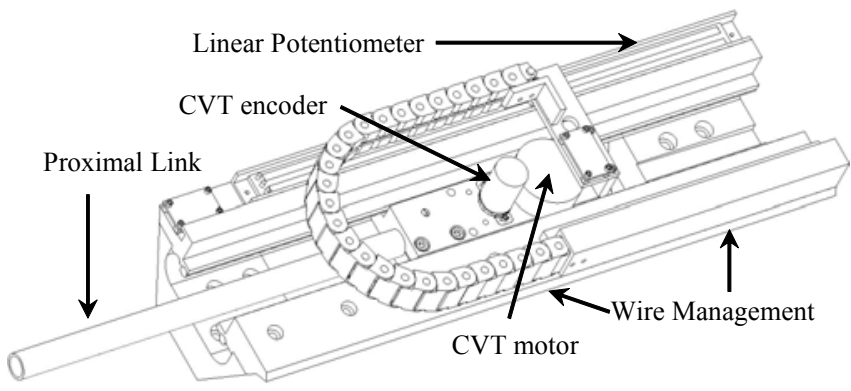

Figure 4. Shown is one of six identical actuator assemblies. The proximal link is grounded to a carraige on a linear guideway. An Igus ${ }^{\mathrm{TM}}$ flexible wire guide manages wiring for the steering motor and encoder.

In Figure 5, the structure of Figure 4 has been inverted and the potentiometer, wire guides, and aluminum structure for the linear guide way removed. Figure 5 details the linear guideway chosen. It was desired to minimize friction and construction tolerances required for the linear guide-way. In addition, it was desired to locate the CVT wheel, which is preloaded against the power cylinder, between the two halves of the linear bearing assembly so as to avoid the generation of significant moments. The resulting design utilizes two guide rods, and five rollers.

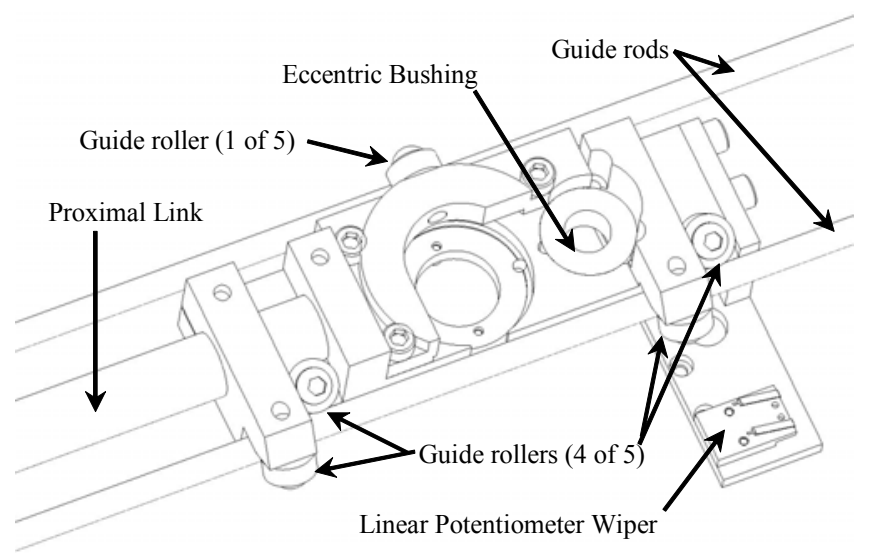

Figure 5. Inverting the assembly in Figure 4 and removing the supporting structure reveals steel guide rods for linear motion. The linear bearing consists of five cam-follower studs, four of which are aligned against one guide rod, the fifth against a second guide rod. The sixth point of contact which constrains the carraige (at least to only one degree of freedom) is provided by the cobotic steering wheel shown in Figure 6. The eccentric bushing allows adjustement of the meshing of the gears shown in Figure 6.

There are several key advantages of this design. Notably, the guide-way operates without binding even when the two guide rods are skew to some extent. But more importantly, the guide-way only works when preloaded by the CVT wheel. As 
the preload at the wheel is increased, the ability of the linear guide way to resist moments on the proximal link increases. This effect goes hand in hand with the desire for the wheel to provide higher constraint forces parallel to the linear motion. An adjustable preload is provided by placing between 9 and 15 Belleville disc springs in some combination of parallel or series between the yoke of the CVT wheel and its housing.

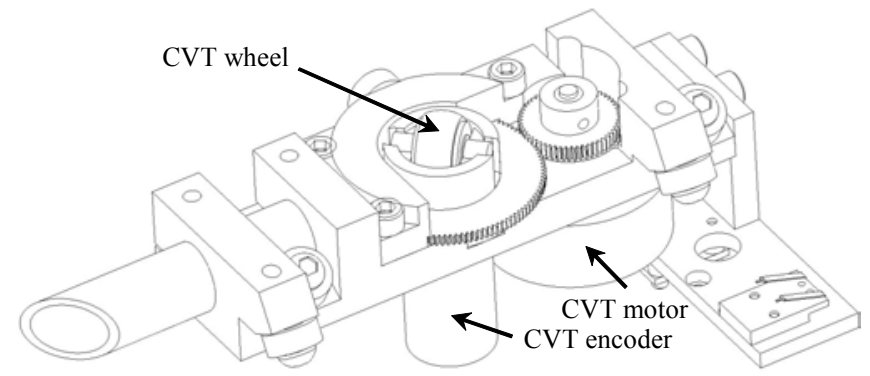

Figure 6. Mounted on the carraige is a steering assembly, consisting of a wheel preloaded via Belleville washers, an optical encoder, a steering motor coupled via gears and a wiper for a linear potentiometer.

Designing wheels for cobots has always been problematic. Conflicting design goals when choosing materials have limited wheel performance. It is desired that the wheels provide a high transverse frictional force with minimal preload, yet it is desired that the wheels have low steering friction to allow for smaller steering actuators and higher bandwidth of control. It is also desired that the wheels have low rolling friction and little dissipation to provide for back-drivability and reduced power requirement for powered cobots. In addition, the wheel should not have any transverse compliance.

Typically, previous cobots have utilized polyurethane Rollerblade $^{\mathrm{TM}}$ wheels in order to obtain the necessary transverse coefficient of friction. As the wheels in this device are $18 \mathrm{~mm}$ in diameter, this was not an option. Since the linear guide-ways already required that a high preload exist, steel wheels were chosen to run against a steel power cylinder even though the coefficient of steel on steel is an order of magnitude less than polyurethane on steel. Thus the need to provide transverse friction and high preload for the linear guide-way are complementary design goals. Depending on performance needs, more or less preload can be utilized. Currently the preload is set around $250 \mathrm{~N}$.

The CVT wheels have a spherical profile and are made of a moderate alloy, around Rockwell C 20. A steel cylinder that has undergone the Nitrotec ${ }^{\mathrm{TM}}$ ion-nitriding process to result in a hardness of Rockwell C 65, yields acceptable wear for each component.

\section{Power Cylinder}

As shown in Figure 7, the power cylinder is located between two mating blocks. The steel cylinder is $25 \mathrm{~cm}$ in length, $14 \mathrm{~cm}$ in diameter, and has a $3.5 \mathrm{~mm}$ wall thickness. Each of the six linear actuator assemblies bolt to the mating blocks. Also connected to the blocks are the power motor and a high resolution encoder. The motor is direct drive to the cylinder with no flexible coupling.

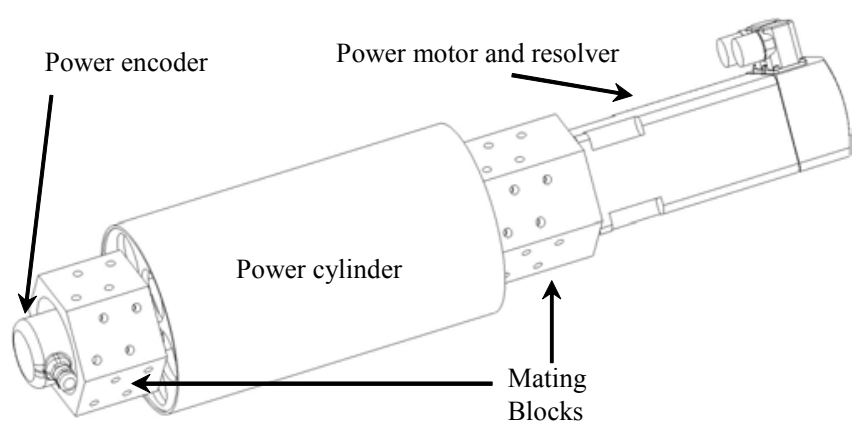

Figure 7. A power motor direct drives the power cylinder.

\section{E. Sensorand ActuatorSpecifications}

Table II summarizes the specifications of the sensors and actuators.

TABLE II. SENSOR AND ACTUATOR SPECIFICATION

\begin{tabular}{|c|c|c|c|c|}
\hline Sensor & Manufacturer & Resolution & Linearity & Range \\
\hline Power Cyl Enc & Gurley & $140,000 \mathrm{cnts} / \mathrm{rev}$ & NA & $2 \pi$ \\
\hline CVT Encoders & Gurley & $40,960 \mathrm{cnts} / \mathrm{rev}$ & NA & $2 \pi$ \\
\hline Linear Pots & ETI & 16 bit ADC $(5 \mu \mathrm{m})$ & $1 / 2000$ & $300 \mathrm{~mm}$ \\
\hline Force-Torque & ATI & 16 bit ADC $(2.5 \mathrm{mN})$ & $1 / 3000( \pm 5 \mathrm{~N}), 1 / 70( \pm 40 \mathrm{~N})$ & $\pm 40 \mathrm{~N}, \pm 2 \mathrm{Nm}$ \\
\hline Actuator & Manufacturer & Peak Torque & Cont Torque & Wattage \\
\hline Power Cyl Motor & Moog & $13 \mathrm{Nm}$ & $3.7 \mathrm{Nm}$ & 1400 \\
\hline CVT Motors & Maxon & $260 \mathrm{mNm}$ & $54 \mathrm{mNm}$ & 30 \\
\hline
\end{tabular}

All motors are brushless DC operated in torque mode. The linear position and force are recorded via 16 bit ADC boards. The linear position signal is differentiated in analog with a two-pole differentiator followed by a Maxim ${ }^{\mathrm{TM}}$ MAX7401 eight-pole Bessel filter-on-chip with programmable cutoff. With this cutoff set at $1000 \mathrm{~Hz}$, and the resulting analog voltage discretized by a 13-bit ADC, linear velocity resolution of $1.0 \mathrm{~mm} / \mathrm{s}$ is achieved for the proximal links at $1000 \mathrm{~Hz}$.

A $1.53 \mathrm{GHz}$ AMD personal computer running QNX 6.2 operates the control system. An oscillator on one of three data acquisition boards is used to generate hardware interrupts at $2500 \mathrm{~Hz}$, to which all data acquisition and output is latched electronically. A Newton optimization scheme computes the forward kinematics. Currently, only one iteration is needed at each control interval to converge to a reasonable residual due to the $2500 \mathrm{~Hz}$ rate relative to the bandwidth of actuation. The inverse Jacobian (36 terms) and the Hessian (216 terms, 90 of which are unique and non-zero) are computed analytically at each time step as well and are required for the transformation from task space to CVT steering velocity space.

\section{PRELIMINARY EXPERIMENTATION}

As describe in the introduction, an operator can interact with the cobot in a "free mode" in the full dimensional sixspace, or while constrained to a one to five dimensional virtualsurface. In Figure 8, a trajectory on a four-dimensional constraint surface is shown. The user's force input in two dimensions is followed via a mass-damping model. The operator is constrained to zero rotation about all three axes, and to the surface of a $17 \mathrm{~cm}$ diameter sphere, which provides a good representation of the available translational workspace. At the center of the Cobotic Hand Controller's translational 
workspace, \pm 40 degree rotations about $\mathrm{x}, \pm 45$ degree rotations about $\mathrm{y}$, and \pm 85 degree rotations about $\mathrm{z}$ are feasible.

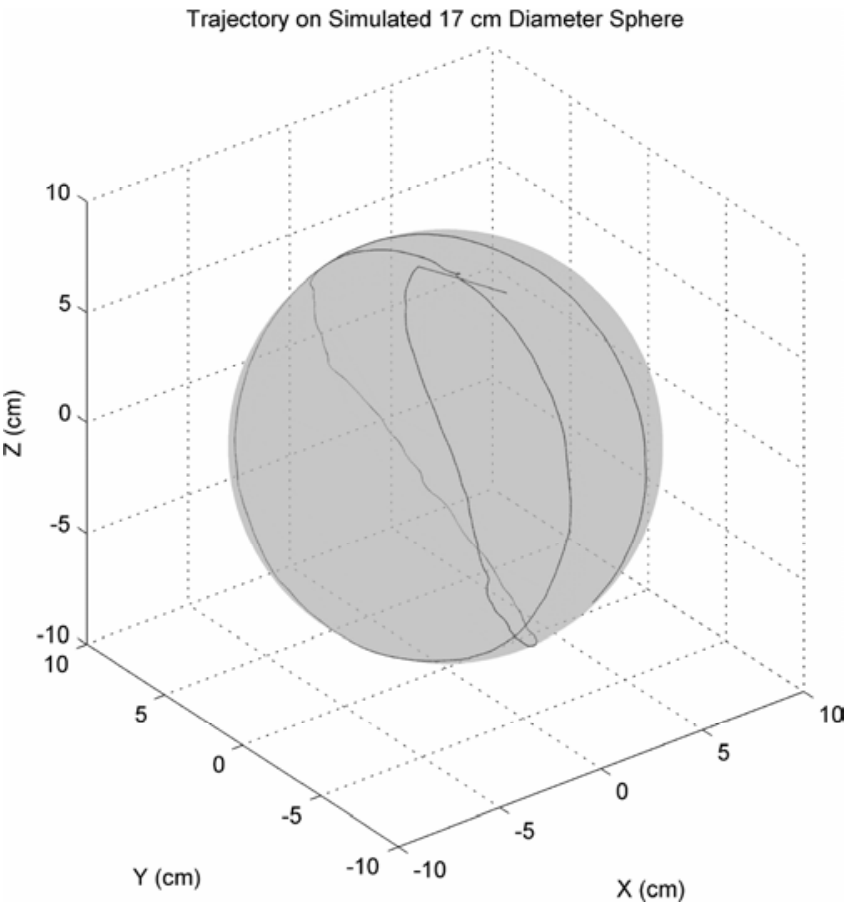

Figure 8. Average tangential force on the user during exploration of the sphere was $0.33 \mathrm{~N}$. Approximately half of this was intentionally due to simulated mass and damping. The surface tracking controller keeps the endeffector to within $80 \mu \mathrm{m}$ of the sphere.

Results from experiments with a second virtual surface are reported in Figures 9 and 10. The figures portray results of a three dimensional constraint in which rotations are constrained to zero but translations are allowed. The translations obey a mass-spring-damper model. The spring constant is $800 \mathrm{~N} / \mathrm{m}$ along the $\mathrm{x}$-axis but $50 \mathrm{kN} / \mathrm{m}$ for excursions in the $\mathrm{y}-\mathrm{z}$ plane. Thus effectively a linear spring along the $\mathrm{x}$-axis is created. Figure 9 shows an operator moving the end-effector back and forth along the $\mathrm{x}$-axis and the incurred force along the $\mathrm{x}$-axis on the operator due to the spring, mass and damper. Figure 10 shows data recorded in the $y-z$ plane during the experiment. The force-displacement curve is only in the first quadrant here, as only the magnitude of force and displacement orthogonal to the $\mathrm{x}$-axis are reported.

Note that these are plots of virtual stiffness and are not absolute measurements incorporating structural stiffness. However, $50 \mathrm{kN} / \mathrm{m}$ was chosen as it is the structural stiffness of the Cobotic Hand Controller in the x-y plane near the center of its workspace. Structural stiffness in $Z$ translation is approximately $400 \mathrm{kN} / \mathrm{m}$ throughout the workspace. Structural stiffness in $\mathrm{x}$ and $\mathrm{y}$ ranges from $50 \mathrm{kN} / \mathrm{m}$ in the center of the workspace to $20 \mathrm{kN} / \mathrm{m}$ away from the $\mathrm{z}$-axis and rotated at extreme angles. The stiffness will go to zero in one or more degrees of freedom when the Jacobian becomes singular.

It is important to note that even while imparting these high stiffness values and forces to the user, the CVT motors require near zero torque and the power cylinder only a few hundred $\mathrm{mNm}$ of torque in order to overcome friction in the device.

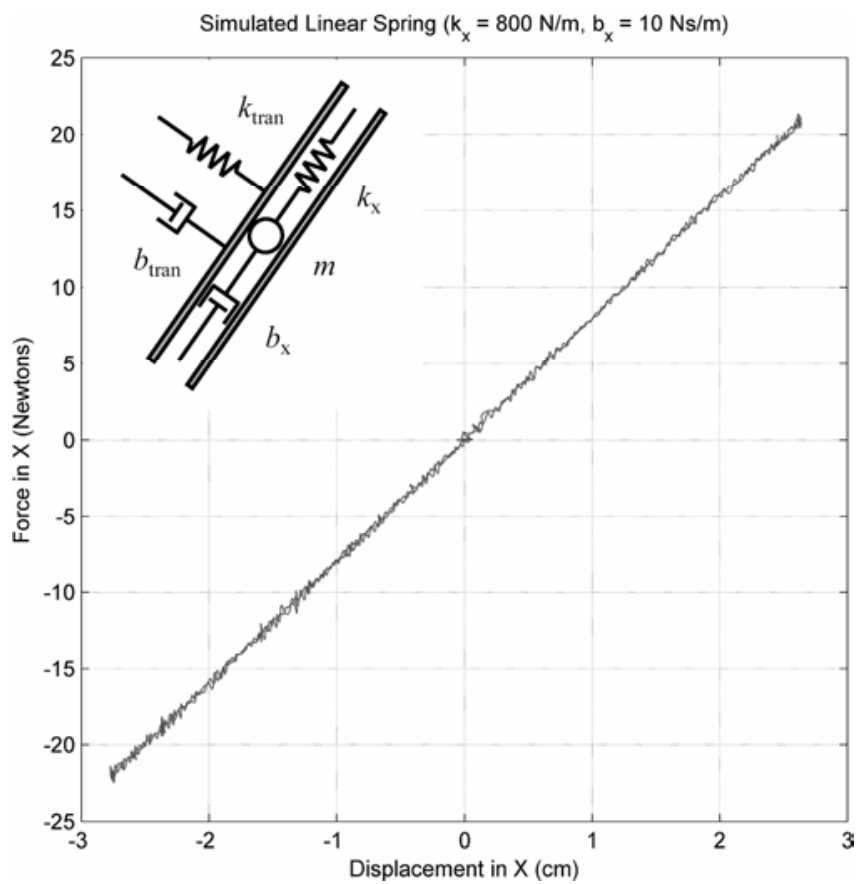

Figure 9. A plot of force versus displacement tangential to a simulated linear spring along the $\mathrm{x}$-axis.

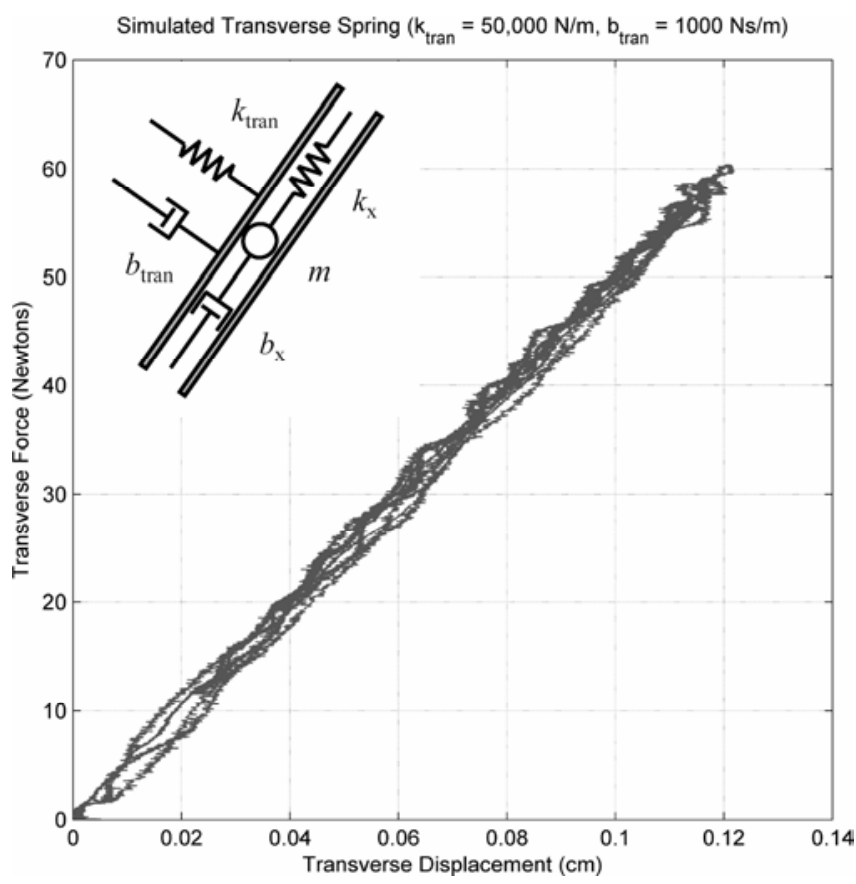

Figure 10. A plot of force versus displacement orthogonal to the $\mathrm{x}$-axis during simulation of a weak $x$-axis linear spring of spring constant $800 \mathrm{~N} / \mathrm{m}$, with a much stiffer transverse spring constant of 50,000 N/m.

\section{CONCLUSION}

An active six-degree-of-freedom Cobotic haptic display with workspace resolution of approximately $25 \mu \mathrm{m}$, force transmission capabilities exceeding $50 \mathrm{~N}$, structural stiffness ranging from $20-400 \mathrm{kN} / \mathrm{m}$ and a translational workspace of a $17 \mathrm{~cm}$ sphere has been designed and built. Based on the 
authors' experience with haptic interface devices, the feeling of this device is quite remarkable. The crisp distinction between free and forbidden directions of motion is striking. This performance arises not from elaborate control algorithms, but from the inherent physical characteristics of the device due to the utilization of non-holonomic constraints.

Many avenues of research lie open for the design of haptic control modes that take advantage of the non-holonomic constraints and the power cylinder. One should note that there is not a unique solution to the angles of the CVTs and the velocity of the power cylinder needed to create a certain task space velocity. It is arbitrary whether to operate the device with the cylinder spinning constantly, even when the endeffector is at rest, or to operate with the cylinder's velocity in some relationship to end-effector velocity. Trade-offs between fidelity, strength, and speed of actuation exist.

Since the ratio of end-effector speed to cylinder speed is arbitrary, it is at the designer's disposal to adjust for various haptic environments and situations. For smooth static surfaces, it may be desired to operate with the cylinder moving slowly relative to end-effector speed, or even rotating at a constant velocity, and thus utilize a wide range of steering angles available to the CVTs. When rendering rough surfaces, it may be desirable to spin the cylinder faster in order to result in larger linear actuator motions with less CVT steering. For dynamic contacts with a regime of high impedance, it may be desirable to adjust the ratio of endpoint speed to cylinder speed with respect to the distance from or velocity relative to the constraint. The Cobotic Hand Controller imparts high impedances not with the strength of actuators, but by the speed at which it can transfer momentum into the power cylinder from the proximal and distal links by steering its CVTs. Thus the power cylinder, or more precisely, "momentum sink," provides a non-electrical means of absorbing a large amount of energy from the user during virtual wall impacts. The mechanism's back-drivability, or lack thereof, is at our control to exploit via the CVTs.

The Cobotic Hand Controller can behave either as a pure admittance device, a pure impedance device, or as a hybrid between an admittance and impedance device. The cobot can be controlled completely as an admittance device if the cylinder rotates at a continuous speed and the CVTs control velocity sources for all motions in six-space. In a pure impedance mode, the torque of the cylinder is controlled and the CVTs are steered such that a model-based force in each proximal link is related to the torque on the cylinder via the steering angles. Alternatively, in a hybrid control mode, the cobot can be operated as an admittance device for motions normal to the current degree of freedom (steering CVTs), and simultaneously as an impedance device along the allowed degree of freedom (closing a force loop tangential to the allowed degree of freedom with the power cylinder).

Although the Cobotic Hand Controller can be controlled as an admittance device, allowing motions based on the applied force, the cobot does not suffer from the high inertia, friction and backlash that normally exist in a highly geared admittance device and is therefore not as limited in the range of impedances it can represent.

\section{ACKNOWLEDGMENTS}

The authors would like to acknowledge the many contributions of their collaborators Young Soo Park, Hyosig Kang and Tom Ewing at Argonne National Labs. The authors would also like to thank Tom Worsnopp and Mark Salada for mechanical design and control issues and Richard Dojutrek and Bob Taglia for assistance in the machine shop.

\section{REFERENCES}

[1] T. B. Sheridan, Telerobotics, Automation, and Human Supervisory Control. Cambridge, MA: MIT Press, 1992.

[2] "Dual arm work platform teleoperated robotics system," Argonne National Laboratory-East, IL, Tech. Rep. DOE/EM-0389, 1998.

[3] M. W. Noakes, L. J. Love, and P. D. Lloyd, "Telerobotic planning and control for DOE D and D operations," in Proc. IEEE International Conference on Robotics and Automation, Washington D.C., 2002, 34853492 .

[4] J. V. Draper, and L.M. Blair, "Dual arm work platform performance estimates and telerobot task network simulation," in Proc. American Nuclear Society Seventh Topical Meeting on Robotics and Remote Handling, Savannah, GA, 1997,

[5] Y. Park, H. Kang, T. F. Ewing, E. F. Faulring, J. E. Colgate, and M. A. Peshkin, "Semi-automatic teleoperation for D\&D," accepted American Nuclear Society 10th International Conference on Robotics and Remote Systems for Hazardous Environments, Gainesville, 2004.

[6] L. B. Rosenberg, "Virtual fixtures: perceptual overlays enhance operator performance in telepresence tasks," Ph.D. dissertation, Stanford University, Stanford, 1994.

[7] P. A. Millman and J. E. Colgate, "Design of a four degree-of-freedom force reflecting manipulandum with a specified force-torque workspace," in Proc. IEEE International Conference on Robotics and Automation, Sacramento, 1991, 1488-1493.

[8] K. Kim, W. K. Chung, and Y. Youm, "Design and analysis of a new 7DOF parallel type haptic device: PATHOS-II," in Proc. IEEE/RSJ International Conference on Intelligent Robots and Systems, Las Vegas, 2003,

[9] V. Hayward et al., "Freedom-7: a high fidelity seven axis haptic device with application to surgical training," in Experimental Robotics V: The Fifth International Symposium, vol. 232, Lecture Notes in Control and Information Science, A. Casalsm and A. T. de Almeida, Eds.: SpringerVerlag, 1998, pp. 445-456.

[10] J. H. Lee, K. S. Eom, B.-J. Yi, and I. H. Suh, "Design of a new 6-DOF parallel haptic device," in Proc. IEEE International Conference on Robotics and Automation, Seoul, Korea, 2001, 886-891.

[11] Y. Tsumaki, H. Naruse, D. N. Nenchev, and M. Uchiyama, "Design of a compact 6-DOF haptic interface," in Proc. IEEE International Conference on Robotics and Automation, Leuven, Belgium, 1998, 25802585 .

[12] (2004, February) Delta Haptic Device. Force Dimension. [Online]. Available: http://www.forcedimension.com/products/files/delta6dof.pdf

[13] (2004, February) Phantom Product Brochure. Sensable Technologies. [Online]. Available: http://www.sensable.com/products/datafiles/ phantom_ghost/PHANTOM_GHOST\%20Brochure.pdf

[14] (2004, February) Virtuose 6D35-45. Haption. [Online]. Available: http://www.haption.com/v3/eng/indexENG.htm

[15] M. A. Peshkin, J. E. Colgate, W. Wannasuphoprasit, C. A. Moore, R. B. Gillespie, and P. Akella, "Cobot architecture," IEEE Transactions on Robotics and Automation, vol. 17, pp. 377-390, 2001.

[16] R. B. Gillespie, J. E. Colgate, and M. A. Peshkin, "A general framework for cobot control," IEEE Transactions on Robotics and Automation, vol. 17, pp. 391-401, 2001.

[17] C. A. Moore, Jr., M. A. Peshkin, and J. E. Colgate, "Cobot implementation of virtual paths and 3D virtual surfaces," IEEE Transactions on Robotics and Automation, vol. 19, pp. 347-351, 2003.

[18] J. P. Merlet, "Direct kinematics and assembly modes of parallel manipulators," International Journal of Robotics Research, vol. 11, pp. 150-162, 1992. 